\title{
OXIDACIÓN DE PROTEÍNAS Y LÍPIDOS EN CEREBRO DE COBAYOS DURANTE LA EXPOSICIÓN A GRANDES ALTURAS (4540 M)
}

\author{
Protein and lipid oxidation in guinea pig brain during acute high altitude exposure (4540 $\mathrm{m}$ ) \\ Anthony M Cárdenas ${ }^{1}$, Luis A Tataje ${ }^{2}$, Edgar A Florentini ${ }^{2}$, Carmen G Peña ${ }^{1}$ y Elizabeth Carranza A ${ }^{1}$ \\ ${ }^{1}$ Instituto Nacional de Biología Andina, Facultad de Medicina, UNMSM. ${ }^{2}$ Facultad de Ciencias Biológicas, UNMSM
}

\section{RESUMEN}

El objetivo del presente estudio fue evaluar el efecto del tiempo de exposición a grandes alturas sobre la oxidación de proteínas y lípidos en el tejido cerebral de cobayos. Se utilizaron cincuenta cobayos nativos del nivel del mar: 1o permanecieron en su hábitat (Lima, $150 \mathrm{~m}$ ) sirviendo de control, y cuarenta fueron trasladados a Morococha, $4540 \mathrm{~m}$, y sacrificados los días 1 , 3, 7 y 14 después de su arribo. Se determinaron: la actividad de fosfolipasa A2 (FLA2), los niveles de hidroperóxidos lipídicos (LOOH) y malondialdehído (TBARS), como marcadores de la oxidación de lípidos; nivel de grupos carbonílicos (GC), como marcadores de la oxidación de proteínas; y la actividad de las enzimas antioxidantes superóxido dismutasa (SOD), catalasa (CAT) y glutatión peroxidasa (GPx). La actividad de FLAz aumentó hasta el tercer día. Se encontró niveles de LOOH, TBARS y GC incrementados al primer día; LOOH mostró tendencia a disminuir durante el experimento; TBARS mantuvo sus niveles altos y GC disminuyó por debajo del nivel de control al tercer día. Las actividades de las enzimas antioxidantes, GPx y CAT, incrementaron desde el primer día; la actividad de SOD aumentó hasta el tercer día y disminuyó posteriormente. Se concluye que la exposición a grandes alturas influye directamente en el proceso de oxidación de lípidos y proteínas. La disminución de los niveles de GC podría deberse a la activación del sistema proteolítico, que degradaría las proteínas dañadas por las EROs (especies reactivas del oxígeno). La exposición a la altura influye además en la actividad de las enzimas antioxidantes, especialmente GPx, por su importante rol en la detoxificación de LOOH.

Palabras clave: oxidación de proteínas, peroxidación lipídica, antioxidantes, altura, cerebro.

\section{SUMMARY}

The aim of this study was to assess the effect of high altitude exposure time on the oxidation of proteins and lipids from guinea pig brain tissue. Fifty guinea pigs (sea level natives) were taken up to Morococha (4540 $\mathrm{m}$ ) and sacrificed on days 1, 3, 7 and 14, after arrival. Phospholipase Az (PLA2) activity, levels of lipid hydroperoxides (LOOH) and malondialdehyde (TBARS) were determined as markers of lipid oxidation. Carbonyl groups (CG) were also determined as markers of protein oxidation. Activities of antioxidant enzymes superoxide dismutase (SOD), catalase (CAT) and glutathione peroxidase (GPx) were also determined. PLA2 activity increased until third day. LOOH, TBARS and CG levels were found to be increased the first day; $\mathrm{LOOH}$ levels showed a declining trend but TBARS levels remained high, CG concentration decreased below the control group levels on the third day. The activities of antioxidant enzymes GPx and CAT increased from the first day; SOD activity increased until the third day and declined afterwards. It can be concluded that exposure to high altitude directly influences the oxidation of lipids and proteins. The decrease in CG levels might be due to proteolytic system activation, which would degrade the proteins damaged by Reactive Oxygen Species (ROS). The altitude exposure also influences the activity of antioxidant enzymes, especially GPx, which plays an important role in the detoxification of LOOH.

Keywords: Proteins oxidation, lipid peroxidation, antioxidants, altitude, brain.

\section{INTRODUCCIÓN}

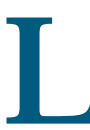

a hipoxia de las GA involucra una serie de respuestas que ocurren en diferentes niveles de organización en el organismo. Así, se produceuna seriederespuestas fisiológicas encaminadas a aumentar el suministro de $\mathrm{O}_{2}$ a los tejidos; a nivel celular se produce liberación de neurotransmisores por las células del glomus del cuerpo carotídeo, secreción de la hormona eritropoyetina, liberación de factores de crecimiento por las células del parénquima en muchos tejidos, aumento en el calcio intracelular, etc.; a nivel molecular hay una expresión inducida de varios genes implicados en la eritropoyesis, la angiogénesis, la remodelación de tejidos, el control vasomotor, la liberación de neurotransmisores, etc., reguladas por la activación de diversos factores de transcripción ${ }^{(1)}$.

La mayoría de las personas que viven a nivel del mar, cuando ascienden a la altura pueden presentar síntomas de mal de montaña agudo, que pueden ser desde trastornos benignos, como ahogo, aumento del ritmo cardíaco, fatiga, dolor de cabeza, náuseas o vómitos e insomnio, hasta 
complicaciones mayores como edema pulmonar y edema cerebral, que pueden producir la muerte ${ }^{(1)}$.

En los últimos años se ha demostrado la asociación entre estrés oxidativo e hipoxia, se ha encontrado un incremento de sustancias reactivas derivadas del oxígeno en modelos de hipoxia/reperfusión (2), ejercicio físico agotador ${ }^{(3)}$, hipoxia simulada en cámara hipobárica ${ }^{(4)}$ y en el ascenso a grandes alturas ${ }^{(5)}$.

El oxígeno, elemento imprescindible para los organismos aerobios para producir energía, puede también alterar los sistemas biológicos a través de una producción incontrolada de radicales libres derivados del oxígeno (EROs), que son capaces de reaccionar con las macromoléculas, dañándolas y alterando los procesos celulares. Las proteínas y lípidos son considerados blanco de la acción de las EROs, al producirse la aparición de grupos carbonílicos (GC), como marcadores de oxidación de proteínas ${ }^{(6)}$, así como hidroperóxidos lipídicos $(\mathrm{LOOH})$ y malondialdehído (MDA) en el proceso de peroxidación lipídica ${ }^{(7)}$.

El cerebro es un órgano sensible al estrés oxidativo debidoasusrequerimientosenergéticosyconsumodeoxígeno elevado, alto contenido de ácidos grasos poliinsaturados que son particularmente vulnerables al ataque de los EROs ${ }^{(8)}$ y un alto contenido de metales de transición como el cobre y el hierro que podrían conducir a la formación de radicales hidroxilo, según la reacción de Fenton ${ }^{(7)}$.

Estudios acerca del efecto de la exposición aguda a las grandes alturas sobre el metabolismo oxidativo cerebral son escasos, por lo que hemos considerado de interés evaluar el efecto de la exposición a las grandes alturas sobre la oxidación de proteínas y lípidos del tejido cerebral de cobayos nativos del nivel del mar trasladados a la ciudad de Morococha (4540 m).

\section{MATERIAL Y MÉTODOS}

\section{Materiales}

El ácido tiobarbitúrico, anaranjado de xilenol, trifenilfosfina (TPP), fosfatidilcolina, Triton X-10o, azul de Coomasie, albúmina, fosfato mono y dipotásico, etilendiamino tetraacético (EDTA), 4-dinitrofenilhidrazina, 2-mercaptoetanol y el ácido tricloroacético fueron adquiridos de Sigma Chemical Company (USA); los kits usados para la determinación de SOD y GPO fueron adquiridos de RANDOX (Reino Unido).

\section{Diseño Experimental}

Se emplearon cincuenta cobayos (Cavia porcellus) adquiridos en el Instituto Nacional de Salud (INS), todos machos adultos nacidos y criados a nivel del mar (Lima, $150 \mathrm{~m}$ ) y alimentados ad-libitum hasta alcanzar un peso promedio de $500 \mathrm{~g}$. Los cobayos fueron separados en cinco grupos de 10 cada uno: Cuatro grupos fueron transportados al laboratorio del Instituto de Biología Andina en Morococha (4540 $\mathrm{m})$, donde permanecieron en un cuarto atemperado a $25^{\circ} \mathrm{C}$, para ser sacrificados luego de $1,3,7$, y 14 días de su arribo. Un grupo, considerado como control, permaneció en el laboratorio del Instituto Nacional de Biología Andina en Lima. Las pruebas analíticas se realizaron en Lima.

Los cobayos fueron decapitados; sus cerebros, retirados por la parte occipital del cráneo, se lavaron con solución de cloruro de sodio $0,9 \%$ a $4^{\circ} \mathrm{C}$ e inmediatamente se guardaron en bolsas de polietileno previamente marcadas, y se congelaron con una mezcla de hielo seco con metanol. Posteriormente se almacenaron a $-35^{\circ} \mathrm{C}$ hasta su procesamiento.

Para el análisis se tomó dos porciones similares de cada cerebro, que luego se homogenizaron en sus respectivas soluciones buffers a $4^{\circ} \mathrm{C}$ por un tiempo no mayor a 3 min.

Para la fracción 1, un gramo de tejido fue homogeneizado con $4 \mathrm{~mL}$ de buffer fosfato salino 5 $\mathrm{mM} \mathrm{pH} \mathrm{7,4,} \mathrm{parte} \mathrm{del} \mathrm{homogeneizado} \mathrm{se} \mathrm{separó} \mathrm{para}$ la determinación de MDA, hidroperóxidos lipídicos y proteínas; el resto fue centrifugado como en el caso anterior y el sobrenadante se utilizó para el análisis de Fosfolipasa A2 y proteínas.

Para la fracción 2, un gramo de tejido fue homogenizado con $10 \mathrm{~mL}$ de una mezcla de buffer fosfato $50 \mathrm{mM} \mathrm{pH}$ 7,o y 2-mercaptoetanol $1 \mathrm{mM}$, parte del homogeneizado se separó para la determinación de grupos carbonilos y de proteínas; el resto fue centrifugado a $10000 \mathrm{rpm}$ a $4^{\circ} \mathrm{C}$ por $30 \mathrm{~min}$, y el sobrenadante se utilizó para el análisis de SOD, GPx, CAT y proteínas.

\section{Determinaciones}

El contenido de GC se determinó según el método descrito por Levine y col. ${ }^{(9)}$, basado en la formación de 2,4-dinitrofenilhidrazona de la reacción entre los grupos carbonílicos de las proteínas oxidadas y el reactivo 2,4-dinitrofenilhidrazina (2,4-DNPH) en medio ácido. Procedimiento: Para la determinación de los GC se tomó $200 \mu \mathrm{L}$ del homogenizado 1 y se añadió $200 \mu \mathrm{L}$ de ácido tricloroacético (ATC) al $20 \%$ a $4^{\circ} \mathrm{C}$ por 5 minutos, se centrifugó a 10000 rpm y al precipitado se le añadió $200 \mu \mathrm{L}$ de 2,4 -DNPH $10 \mathrm{mM}$ en $\mathrm{HCl}_{2} \mathrm{M}$, se 

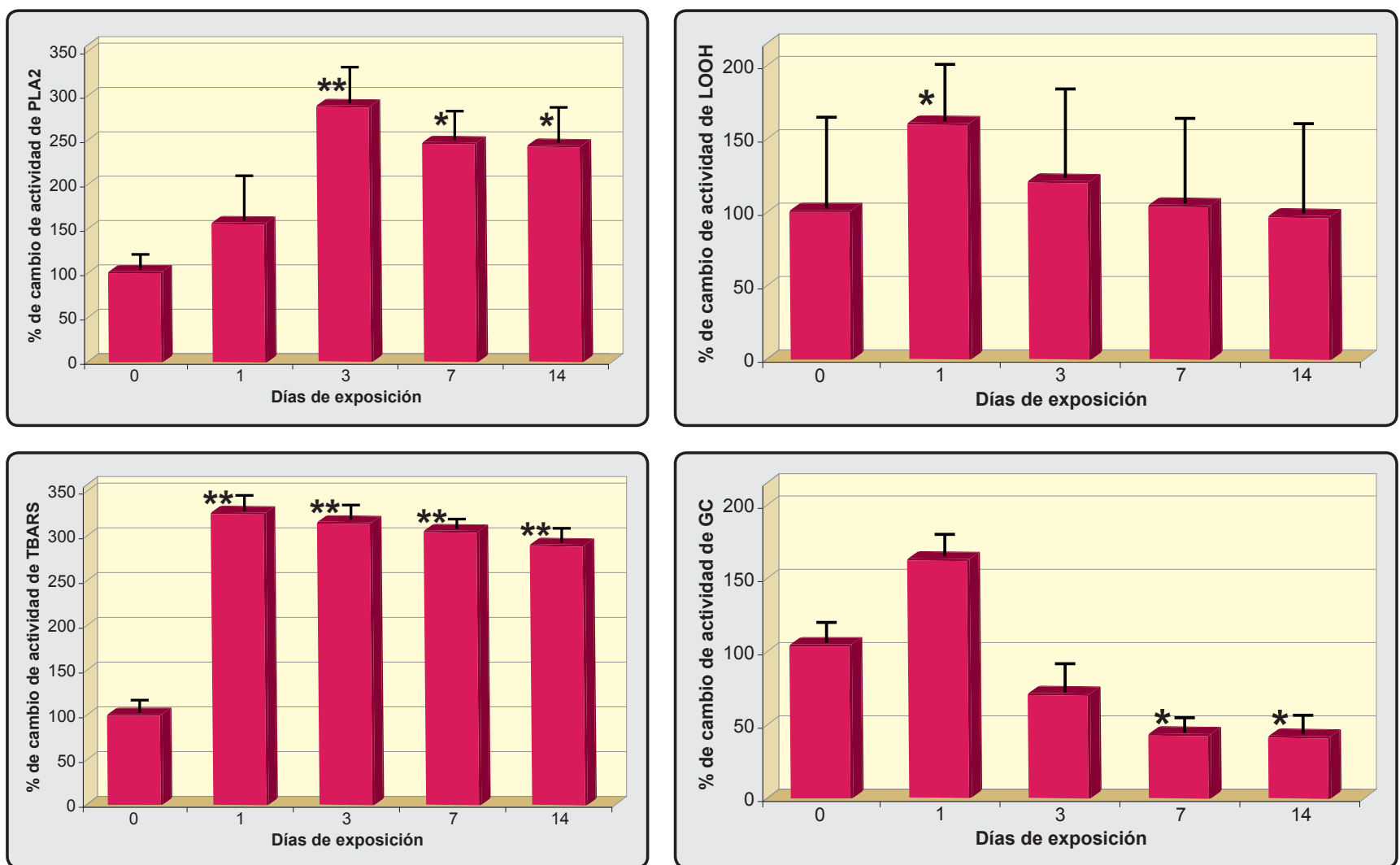

Figura 1. Porcentaje de cambio de la actividad de la FLA2 y niveles de LCOOH, TBARS y GC durante la exposición a las grandes alturas (4300 $\mathrm{m})$. Los resultados son expresados como medias. La media del grupo 0 corresponde al $100 \%$.

* $p<0,05$ respecto del grupo $0 \quad{ }^{* *} p<0$, ool respecto del grupo 0

incubó por 30 min con agitación cada 5 min. Después, se añadió $200 \mu \mathrm{L}$ de ATC $50 \%$ e inmediatamente se centrifugó en refrigeración a $4000 \mathrm{rpm}$ por $10 \mathrm{~min}$. Se descartó el sobrenadante y el pellet se lavó 3 veces con $500 \mu \mathrm{L}$ de Etanol:Acetato de etilo (1:1). Finalmente, el pellet se resuspendió en solución de guanidina 6 My fue leído por espectrofotometría a 270 ๆm. Paralelamente, se preparó el blanco correspondiente con $\mathrm{HCl}_{2} \mathrm{M}$. Para los cálculos, se trabajó con la diferencia de absorbancia entre la muestra y su respectivo blanco, y se utilizó el coeficiente de extinción de $22000 \mathrm{M}^{-1} \mathrm{~cm}^{-1}$ para la 2,4-dinitrofenilhidrazina a $270 \eta \mathrm{m}$. Los resultados se expresaron como ๆmoles de $\mathrm{GC} / \mathrm{mg}$ de proteína.

Los niveles de hidroperóxidos lipídicos ( $\mathrm{LOOH})$, de malondialdehido (MDA) y de la actividad de Fosfolipasa A2, superóxido dismutasa (SOD), catalasa (CAT) y glutation peroxidasa (GPx), se determinaron según los protocolos descritos previamente ${ }^{(10)}$. El nivel de MDA se expresa como "Sustancias Reactivas con el Acido Tiobarbiturico" (TBARS). El contenido de proteínas para GC fue medido por el método espectrofotométrico UV (a 280 ๆm), y para los demás parámetros por el método de Bradford, usando albúmina sérica bovina como estándar ${ }^{(10)}$.

\section{Análisis estadístico}

Se efectuó la comparación de medias mediante el análisis de varianza. Para los resultados con varianzas homogéneas se aplicó la prueba de Tukey y para aquellos con varianzas heterogéneas la prueba de Tamhane. Se consideró significativo todo resultado cuyo valor asociado de $p$ fuese $<0,05$. El análisis estadístico se llevó a cabo mediante el programa SPSS versión 15,0.

\section{RESULTADOS}

Todos los resultados se presentan como porcentaje de variación, en relación al grupo control (o). A la media de este grupo se tomó como el 100\%.

En la figura 1 se muestra las variaciones, expresadas en porcentajes de la actividad enzimática de $\mathrm{FLA}_{2}$ y de los niveles de LOOH, TBARS y GC.

En relación a la enzima $\mathrm{FLA}_{2}$, se observa un incremento de la actividad hasta el tercer día de 
exposición a la altura, el cual se mantiene los días 7 y 14. Existe diferencia de los grupos de 3, 7 y 14 días de exposición a la altura con relación al grupo control $(p<0,05)$, pero no entre ellos mismos. Los niveles de $\mathrm{LOOH}$ aumentan el primer día de exposición a la altura $(p<0,05)$, disminuyen desde el $3^{\text {er }}$ día, pero sin diferencia significativa con relación al control ni entre éstos. Los niveles de TBARS incrementaron desde el primer día de exposición, los cuales se mantienen hasta el día 14, presentando todos los grupos diferencia con respecto al control $(p$ $<0,05)$, no presentando diferencia significativa entre ellos. La concentración de GC al primer día de exposición aumentó sin diferencia significativa con relación al grupo control. A partir del tercer día hay una disminución de las concentraciones de GC por debajo de los niveles del control, sólo los grupos de los días 7 y 14 presentaron diferencia en relación al control ( $\mathrm{p}$ $<0,05)$. No existe diferencia significativa entre los grupos expuestos a la altura.

Los resultados de la estimación de la actividad enzimática antioxidante evidencia un incremento de la SOD al primer y tercer día de exposición; sin embargo, al $7^{\text {mo }}$ día dichos valores disminuyen al nivel del grupo control manteniendo la tendencia hasta el día 14. Sólo hay diferencia $(p<0,05)$ entre el grupo del $3^{\text {er }}$ día con relación al grupo control. No existe diferencia significativa de las actividades de SOD entre los grupos expuestos a la altura; se encontró un aumento de la actividad de la enzima CAT en los diferentes tiempos de estudio, siendo los grupos de los días 1, 7 y 14 estadísticamente diferentes respecto al control $(p<0,05)$; sin embargo, no existe diferencia significativa entre las actividades enzimáticas de los grupos expuestos a altura. Finalmente, la enzima GPx muestra un incremento exponencial de su actividad en relación al tiempo, existiendo diferencia entre los grupos de los días 7 y 14 con respecto al grupo control $(p<0,05)$ y también entre los grupos de los días 1, 3 y 7 con relación al grupo del día 14 de exposición $(p<0,05)$, tal como se muestra en la figura $\mathrm{N}^{\circ} 2$.

\section{DISCUSIÓN}

La exposición aguda a la altura está relacionada con un aumento de las EROs, lo cual es explicado por
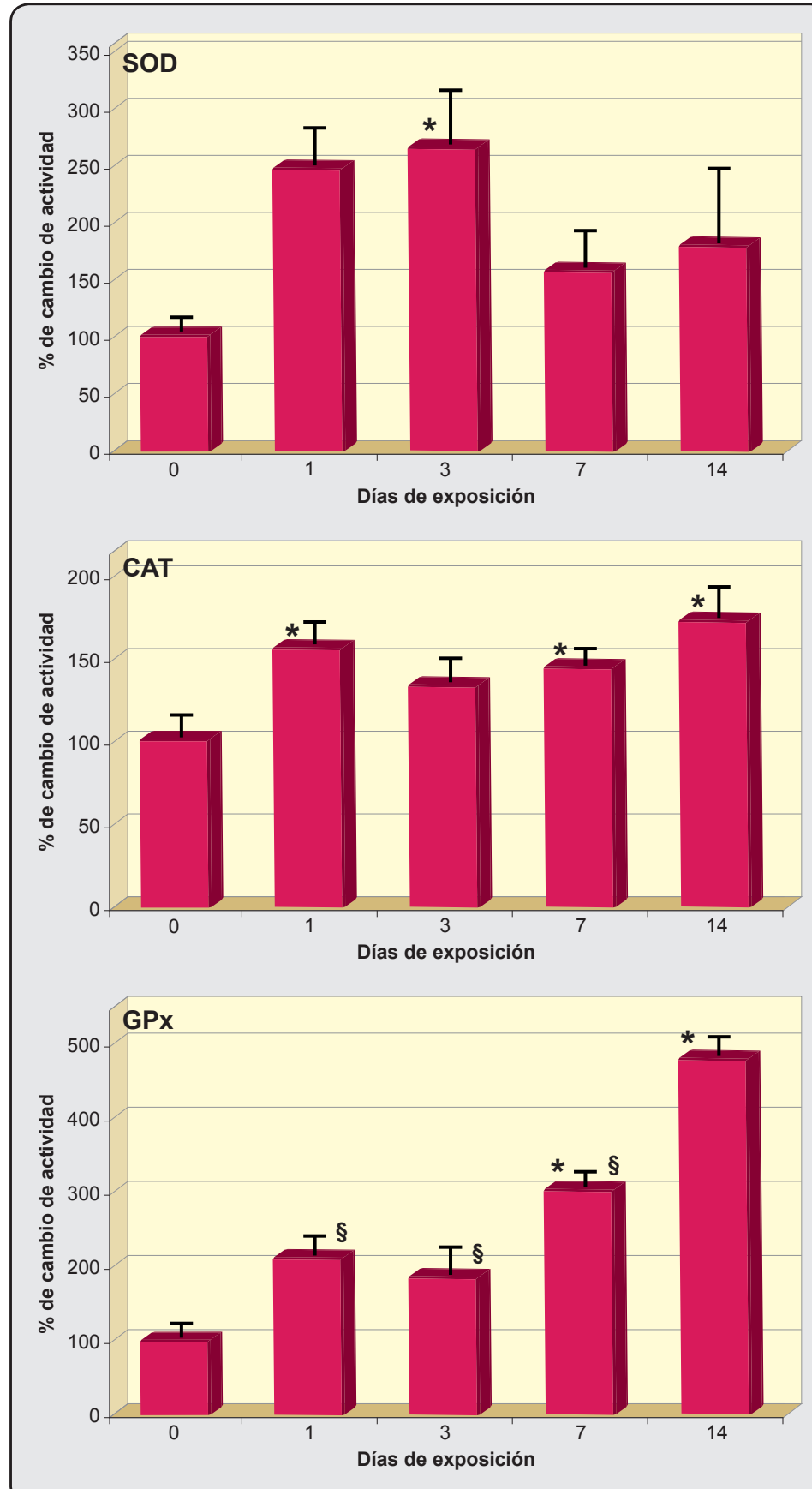

Figura 2. Porcentaje de cambio de la actividad de Las enzimas SOD, CAT y GPx durante la exposición a las grandes alturas (4 $300 \mathrm{~m}$ ). Los resultados son expresados como medias. La media del grupo 0 corresponde al $100 \%$.

* $p<0,05$ respecto del grupo $0 \quad \S p<0$,05 respecto del grupo 14 días

diferentes mecanismos, entre ellos los relacionados directamente al aumento del $\mathrm{Ca}^{+2}$ intracelular y al mayor consumo de ATP. En condiciones normales el ATP es metabolizado hasta xantina para ser convertido a ácido úrico por acción de la xantina deshidrogenasa; 
sin embargo, altas concentraciones de $\mathrm{Ca}^{+2}$ intracelular estimulan la conversión de la xantina deshidrogenasa a xantina oxidasa, que metaboliza la misma reacción pero con la liberación de un radical superóxido $\left(\mathrm{O}_{2}{ }^{--}\right)^{(11)}$. Seha reportado un aumento de los niveles de catecolaminas en sangre, lo que conlleva a un aumento de la produccción de EROs generadas por su proceso de autooxidación (12). Asimismo, numerosos estudios demuestran un incremento de la producción de indicadores de estrés oxidativo en músculo, sangre, orina y en diferentes tejidos en respuesta a la hipoxia ${ }^{(2-4)}$. Existen escasos estudios relacionados al metabolismo oxidativo en el tejido cerebral en la exposición a grandes alturas usando modelos animales y, menos aún, que hayan realizado un seguimiento continuo del sistema antioxidante y de los marcadores de estrés oxidativo. Frente al incremento de la producción de EROs, la primera línea de defensa son las enzimas antioxidantes constituidas por SOD, CAT y GPx; que actúan reduciendo la velocidad de iniciación de la cadena oxidativa ${ }^{(13)}$.

En el presente estudio se encontró que los niveles de las actividades de las enzimas mencionadas en tejido cerebral de cobayos nacidos y criados al nivel del mar, se incrementan desde el primer día de exposición a las grandes alturas $(4540 \mathrm{~m})$, alcanzando actividades máximas al tercer día para SOD y al día 14 para CAT y GPx; sin embargo, los resultados demuestran que SOD no jugaría un rol muy importante en el sistema antioxidante, pues su actividad disminuye a partir del tercer día hasta alcanzar actividades similares a las del nivel del mar. Esto es debido a que esta enzima es inhibida por retroalimentación, no así con CAT ni con GPx, cuyas actividades se fueron incrementando durante los 14 días de estudio. CAT y GPx se encargarían de catalizar la formación de $\mathrm{H}_{2} \mathrm{O}$ a partir de $\mathrm{H}_{2} \mathrm{O}_{2}$; sin embargo, las altas concentraciones de hierro cerebral, que pueden alcanzar hasta valores de $21,3 \mathrm{mg} / 100 \mathrm{mg}$, favorecen la reacción de Fenton y Haber-Weiss y la formación de radicales $\mathrm{OH} \bullet$, radical altamente reactivo que ocasiona y da inicio a la oxidación de proteínas y lípidos ${ }^{(13)}$, lo que justificaría nuestros resultados relacionados a los altos niveles de GC e hidroperóxidos lipídicos encontrados el primer día de exposición a la altura.

En el presente estudio es evidente un incremento en la formación de GC como marcador de la oxidación de proteínas al primer día de exposición, lo que concuerda con Sahin y col, quienes reportan similar incremento de GC en cerebro de ratas en dos modelos de estrés: por frío e inmovilización ${ }^{(14)}$. Los niveles de GC disminuyeron desde el día 3 al 14 por debajo del $65 \%$ de los niveles del grupo control. Radak y col. reportaron también una disminución del $26 \%$ del nivel de GC en el cerebro de ratas expuestas durante 4 semanas a $4000 \mathrm{~m}$ de altura simulada en cámara hipobárica ${ }^{(15)}$. Para explicar las diferencias en la disminución de GC en ambos estudios, deben tomarse en cuenta: el mayor tiempo de exposición, la diferencia de los modelos experimentales, y el hecho de que en la altura influirían otros factores diferentes a la menor presión parcial de $\mathrm{O}_{2}$.

La disminución de GC indicaría una disminución de la producción de EROs; sin embargo, se ha demostrado que la exposición a grandes alturas disminuye la velocidad de síntesis de proteínas y posiblemente incremente su degradación al activar los sistemas proteolíticos. Así, la disminución de GC podría justificarse por el aumento en la concentración de $\mathrm{Ca}^{+2}$ intracelular en la neurona, que activaría a las proteasas dependientes de calcio:calpaínas ${ }^{(16)}$, la cuales las degradarían hasta sus unidades aminoacídicas, para luego ser reutilizadas en el sistema de recambio proteico. Por otro lado, las proteínas alteradas podrían ser ubiquitinizadas, convirtiéndose en blanco del sistema proteolítico no lisosomal o proteasomal, que también las degradaría hasta aminoácidos.

En el proceso de peroxidación lipídica, los niveles de $\mathrm{LOOH}$ se incrementan desde el primer día de exposición con diferencia significativa en relación al grupo control; a partir del día 3, estos niveles descienden drásticamente con tendencia a seguir disminuyendo en el tiempo. Sin embargo, los niveles de TBARS incrementan sus niveles al primer día de exposición, no variando con el tiempo, y en todos los casos se obtuvo diferencia significativa al compararse con el grupo control.

El incremento de los niveles de LOOH y TBARS en el $1^{\text {er }}$ día, podría ser explicado por un incremento de la actividad de FLA2 también desde el $1^{\text {er }}$ día, mediado por el aumento de $\mathrm{Ca}^{+2}$ intracelular, que liberaría una mayor cantidad de ácidos grasos de la membrana, susceptibles al ataque de las EROs, dando inicio a la cadena de peroxidación lipídica. Sin embargo, la acumulación de TBARS no guarda relación con la disminución de la formación de $\mathrm{LOOH}$, debido quizás al rol protector que cumple la GPx al catalizar la formación de alcoholes lipídicos a partir de LOOH. En el presente estudio se encontró un incremento significativo de la actividad de GPx desde el día 1 al 14, lo cual justificaría la disminución de los niveles de LOOH debido a que éstos pasaron a su forma alcohólica. 


\section{CONCLUSIONES}

Los resultados obtenidos en el presente estudio nos permiten concluir que durante la exposición a las grandes alturas:

1. Se produce un incremento del estrés oxidativo en tejido cerebral y que la lipoperoxidación y la oxidación de proteínas difieren en sus repuestas, pues mientras que el TBARS incrementó durante toda la exposición a la hipoxia de altura, los niveles de los grupos carbonilos sólo se incrementaron el primer día.

2. La hipoxia de las grandes alturas influye en forma diferente en las enzimas antioxidantes: se observa una disminución de la actividad de SOD, y una mayor actividad de CAT y GSH-Px que va a permitir a la célula descomponer hidroperóxidos y evitar de esta manera la reacción de Fenton.

\section{REFERENCIAS BIBLIOGRÁFICAS}

1. West JB, Schoene RB, Milledge JS. High altitude medicine and physiology. $4^{\mathrm{a}}$ ed. Hodder Arnold a Member of the Hodder Headline Group. London, 2007.

2. Milei J, Forcada P, Fraga CG, Grana DR, Iannelli G, Chiarello M, et al. Relationship between oxidative stress, lipid peroxidation, and ultra-structural damage in patients with coronary artery disease undergoing cardioplegic arrest/reperfusion. Cardiovasc Res 2007; 73(4):710-9.

3. Fisher-Wellman K, Bloomer RJ. Acute exercise and oxidative stress: a 30 year history. Dyn Med 2009; 8: 1. [En línea] Acceso o6 octubre 2012. Disponible en: http:// www.ncbi.nlm.nih.gov/pmc/articles/PMC2642810/ pdf/1476-5918-8-1.pdf).

4. Magalhães J, Ascensão A, Soares J, et al. Acute and severe hypobaric hypoxia increases oxidative stress and impairs mitochondrial function in mouse skeletal muscle. J Appl Physiol 2005; 99(4): 1247-53.

5. Sinha S, Ray US, Tomar OS, Singh SN. Different adaptation patterns of antioxidant system in natives and sojourners at high altitude. Respir Physiol Neurobiol 2009; 167(3): 255-6o.

6. Cecarini V, Gee J, Fioretti E, et al, Protein oxidation and cellular homeostasis: Emphasis on metabolism. Biochim Biophys Acta 2007; 1773: 93-104.

7. Niki E, Yoshida Y, Saito Y, Noguchi N. Lipid peroxidation: mechanisms, inhibition, and biological effects. Biochem Biophys Res Commun 2005; 338: 668-76
8. Mancinelli R, Barlocci E, Palminiello S and Saso L. Oxidative stress and brain deseases: Biomarkers and analytical methodologies. Indian J Biotechnol 2011; 10: 395-403.

9. Levine RL, Garland D, Oliver CN, Amici A, Climent I, Lenz AG, et al. Determination of carbonyl content in oxidatively modified proteins. Methods Enzymol 1990; 186: 464-78.

10. Pajuelo M, Yamada L, Florentini E, Carranza E. Enzimas antioxidantes en cerebro de cobayos nativos de las grandes alturas. Ciencia e Investigación 2004; 7(2): 18-23.

11. Brown JM, Terada LS, Grosso MA, Whitmann GJ, Velasco SE, Patt A, et al. Xanthine oxidase produces hydrogen peroxide which contributes to reperfusion injury of ischemic, isolated, perfused rat hearts. J. Clin. Invest. 1988; 81: 1297-1301.

12. Oyola L, Zúñiga H, Carranza E, Florentini E. Catecolaminas en sujetos expuestos a hipoxia aguda y crónica. Anales de la Facultad de Medicina 2002; 63 (Suppl): 36 .

13. Matés J, Pérez-Gómez C, Nuñez de Castro I. Antioxidant enzymes and human diseases. Clin Biochem 1999; 32: 595-603.

14. Sahin E, Gümüşlü S. Alterations in brain antioxidant status, protein oxidation and lipid peroxidation in reponse to different stress models. Behavioural Brain Research 2004; 155: 241-8.

15. Radak Z, et al. Oxidative stress induced by intermittent exposure at a simulates altitude of $4000 \mathrm{~m}$ decreases mitochondrial superoxide dismutase content in soleus muscle of rat. Eur J Appl Physiol Occup Physiol) 1994; 69(5): 392-5.

16. Croall DE, DeMartino GN. Calcium-Activated Neutral Protease (Calpain) System: Structure, Function, and Regulation. Physiol Rev 1991; 71(3): 813-47.

Manuscrito recibido el: 06/11/2012

Aceptado para su publicación el: 10/12/2012

\section{Correspondencia:}

Nombre: Elizabeth Carranza Alva

Dirección: Instituto Nacional de Biología Andina

Facultad de Medicina - UNMSM.

Av. Alfonso Ugarte 88o. Lima 1, Perú.

e-mail: ecarranzalva@yahoo.com 